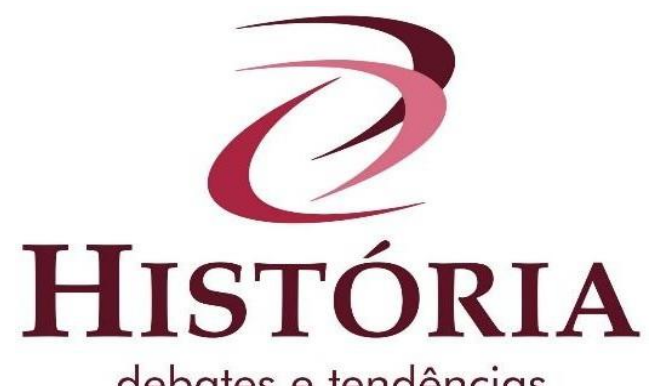

debates e tendências

\title{
Matrices teórico-políticas del pensamiento de Sonia Fleury y la economía política de la salud en los años 1980 en Brasil
}

\section{Matrizes teórico-políticas do pensamento de Sonia Fleury e a economia política de saúde na década de 1980 no Brasil}

\section{Theoretical-political matrices of Sonia Fleury's thought and the political economy of health at 1980s in Brazil}

\author{
Leonardo Carnut ${ }^{\mathrm{i}}$ \\ Aquilas Nogueira Mendes ${ }^{\text {ii }}$
}

\begin{abstract}
Resumen: La Reforma Sanitaria Brasileña (RSB) se inserta en el contexto de los años 1980 en Brasil como un movimiento con base técnica e intelectual fuerte y fuerza social pujante. Una de sus intelectuales, Sonia Fleury, fue una militante y investigadora brasileña cuyo trabajo destacado en la Asamblea Nacional Constituyente de 1988 para la elaboración del Sistema Único de Salud (SUS) le dió notoriedad. Por eso, en memoria a su legado, realizamos un análisis de las matrices teórico-políticas que Fleury elabora para pensar la economía política de la salud en este contexto. Para esta tarea hicimos un análisis de contenido, de tipo temático-evaluativa, de dos obras de esta autora del período postconstituyente (1988-1992): "Reforma sanitaria: En busca de una teoría" (1989) y "Estado y politicas sociales en América Latina" (1992), basándose en Quivy y Campenhoudt. Optamos por la modalidad textual de ensayo crítico como manera de elaborar mejor el pensamiento. Dos grandes bloques de análisis pudieron ser identificado en las obras. Uno relacionado al Estado como centro del debate y la coyuntura de los 1980, y otro que descortina las matrices teórico-políticas y su originalidad para aquella escena históricopolítica. Por ende, rescatar Sonia Fleury y su aporte téorico es muy importante para no que se pierda la historia política sobre las categorías de la economía política de una lucha por el derecho a la salud en sociedades capitalistas como la nuestra.
\end{abstract}

Palabras claves: Historia; Teoría Política; Medicina Social, Sistema Único de Salud, Investigación Cualitativa.

Resumo: A Reforma Sanitária Brasileira (RSB) está inserida no contexto da década de 1980 no Brasil como um movimento com forte base técnica e intelectual e uma força social pujante. Uma de suas intelectuais, Sonia Fleury, era uma ativista e pesquisadora brasileira cujo destacado trabalho na Assembleia Nacional Constituinte de 1988 para a elaboração do Sistema Único de Saúde (SUS) lhe deu notoriedade. Por isso, em memória de seu legado, realizamos uma análise das matrizes teórico-políticas que Fleury elabora para pensar a economia política da saúde nesse contexto. Para tanto, realizamos uma análise de conteúdo, de tipo temático-avaliativo, de duas obras desta autora do período pós-constitucional (19881992): "Reforma sanitária: em busca de uma teoría" (1989) e "Estado e política sociais na América Latina" (1992), baseado em Quivy e Campenhoudt. Optamos pela modalidade textual de ensaio crítico como forma de melhor elaborar o pensamento. Dois grandes blocos 
de análise puderam ser identificados nas obras. Uma relacionada ao Estado como centro do debate e da situação dos anos 1980, e outra que desvela as matrizes teórico-políticas e sua originalidade para aquele cenário histórico-político. Portanto, resgatar Sonia Fleury e sua contribuição teórica é muito importante para não perder a história política das categorias da economia política de luta pelo direito à saúde em sociedades capitalistas como a nossa.

Palavras-chave: História; Teoria Política; Medicina Social, Sistema Único de Saúde, Pesquisa Qualitativa.

\begin{abstract}
The Brazilian Health Reform (RSB) is inserted in the context of the 1980s in Brazil as a movement with a strong technical and intellectual base and a thriving social force. One of its intellectuals, Sonia Fleury, was a Brazilian activist and researcher whose outstanding work in the 1988 National Constituent Assembly for the elaboration of the Unified Health System (SUS) gave her notoriety. For this reason, in memory of her legacy, we carried out an analysis of the theoretical-political matrices that Fleury elaborates to think about the political economy of health in this context. For this task, we made a content analysis, of a thematic-evaluative type, of two works by this author from the postconstitutional period (1988-1992): "Health reform: In search of a theory" (1989) and "State and politics Social in Latin America "(1992), based on Quivy and Campenhoudt. We opted for the textual modality of critical essay as a way to better elaborate thought. Two large blocks of analysis could be identified in the works. One related to the State as the center of debate and the situation of the 1980s, and another that uncovers the theoretical-political matrices and its originality for that historical-political scene. Therefore, rescuing Sonia Fleury and her theoretical contribution is very important so as not to lose the political history on the categories of political economy of a fight for the right to health in capitalist societies like ours.
\end{abstract}

Keywords: History; Political Theory; Social Medicine, Unified Health System, Qualitative Research.

\title{
Introducción
}

Los procesos de transición política siempre son buenos momentos para se observar fenómenos históricos así como la interpretación que sus actores dan a la escena vivida, especialmente en la salud (VILAR-RODRÍGUEZ y PONS-PONS, 2018). Un ejemplo de este aspecto fue lo que se vivenció en Brasil. Con el proceso de redemocratización en la transición del régimen político de la dictadura cívico-militar-empresarial a la Nueva República en Brasil (1985-1988), las diversas luchas sociales demandaron desde la centralidad de la cuestión social una búsqueda de la legitimación de la orientación democrático-popular. Entre estas luchas, la Reforma Sanitaria Brasileña (RSB) se inserta en este contexto como un movimiento con una base técnica e intelectual (PAIM, 2008), principalmente representado por el trabajo de los sanitarios afiliados al Centro Brasileño de Estudios en Salud (Cebes) y la Asociación Brasileña de Salud Coletctiva (Abrasco), y, en cierta medida, asociados a una base popular ejemplificada por el trabajo del Movimiento Popular en Salud (Mops) (DANTAS, 2018), Movimientos de Base Eclesiales y varios grupos más pequeños de trabajadores y sociedad civil organizados de manera autónoma. 
Una de las particularidades del movimiento de Reforma Sanitaria fue, en cierta medida, el uso de categorías centrales de la tradición marxista y la acción de inspiración socialista para componer la comprensión de la situación política en la que se encontraban. El uso de estas categorías contribuyó en gran medida a profundizar el análisis del contexto brasileño de lucha de clases en el que se encontraban los reformistas en este período de transición de un régimen militar hacia la democratización.

Mientras tanto, tradicionalmente, existe una visión romántica, e incluso nostálgica, del Movimiento de Reforma Sanitaria como fuente de inspiración socialista que poco a poco se fue perdiendo durante la implementación del Sistema Único de Salud (SUS). Este sistema de salud fue la consequencia práctica de la lucha de los trabajadores, intelectuales, partidos y movimientos sociales que deseaban cambios profundos en el sistema de salud. La principal consigna era garantizar la salud como un derecho social positivado constitucionalmente en este proceso de transición. Pero, después de la conquista políticojurídica haber sido ganada, la implantación del SUS (de 1988 hasta hoy) siempre se ve amenazada político-economicamente.

Desde de su implantación la tesis socialista (que inspiraba los sanitaristas) fuera abandonada en nombre de una ideología política socialdemócrata (PAIM, 2008; OLIVEIRA, 1987; AROUCA, 2003). En este sentido, la apuesta táctico-estratégica se enfocó en la ocupación institucional. Si bien que la RSB es un hecho histórico con varios autores-actores (PAIVA y TEIXEIRA, 2014), se considera que, para fines didácticopedagógicos, se debe realizar la elección de representantes y su participación para la comprensión de la elección política tomada por los reformistas. En este sentido, a los efectos de este artículo, se prioriza el estudio de textos específicos Sonia Maria Fleury Teixeira (más conocida como Sonia Fleury), una autora-actriz considerada como una de los exponentes que representan las contradicciones vividas en la corriente del pensamiento político en esta arena.

Sonia Fleury es una importante formuladora de la Reforma Sanitaria brasileña que se basa en el método marxista de intervención política de Gramsci y, hasta el día de hoy, continúa actuando como intelectual en el campo de la Salud Colectiva ${ }^{i i i}$ en Brasil. Fleury empezó su trabajo en el curso de Psicología Social de la Universidade Federal de Minas Gerais (UFMG), bajo la coordinación del Profesor Célio García, donde desarrolló actividades de docencia, investigación y análisis institucional durante la década de 1970. En este período participó de análisis institucionales conjuntuamente con Michel Foucault y Georges Lapassade. 
A finales de los años 1970, trabajó en el Financiador para Estudios y Proyectos (FINEP) del Ministerio de Ciencia y Tecnología en Brasil, donde participó en la implantación del programa de apoyo a investigaciones que ayudó a constituir el campo de la Salud Colectiva ${ }^{\text {iv }}$. Al mismo tiempo, actuó como investigadora del Programa de Estudios Socioeconómicos de Salud (PESES) en parceria con el FINEP y la Fundación Oswaldo Cruz (FIOCRUZ) bajo la coordinación de Sergio Aroucav ${ }^{v}$, analizando el Complejo Previsional de Asistencia. Participó activamente en la lucha por la democratización como liderazgo en algunas de las más actuantes instituciones del sector de salud como el Centro de Estudo Brasileños en Salud (Cebes) y la Asociación Brasileña de Salud Colectiva (Abrasco), donde fue formulado e impulsado el proyecto de la Reforma Sanitaria Brasileña que resultó en la creación del Sistema Único de Salud (SUS).

Mientras el período de la Nueva República en Brasil actuó como consultora del Ministerio de la Jubilación Social, en la gestión del Dr. Waldir Pires, donde se formuló la propuesta de una previsión viable e inclusiva. También fue miembra del consejo consultor del Instituto Brasileño de Análisis Social y Económico (IBASE) durante la gestión de Betinho, y sobre la participación política en organizaciones de la sociedad civil ${ }^{\mathrm{vi}}$ refundó el Cebes como presidente en la gestión 2006-2009.

La experiencia de especialista associada a de militante en el área la calificó para ejercer el papel de consultora de la Asamblea Nacional Constituyente para la elaboración del capítulo sobre la Seguridad Social de la Constitución brasileña de 1988, en la relatoría del Senador Almir Gabriel. En el marco de la Conferencia de las Naciones Unidas sobre el Cambio Climático, ella realizó una revisión de la propuesta de la Comisión Sanitaria Brasileña.

En la presidencia de la Fiocruz, Fleury asesoró al vicepresidente, Mario Hamilton en el desarrollo de la metodología e implantación del $1^{\circ}$. Congreso Interno de la Fiocruz, una experiencia original de participación en la planificación institucional. Se retiró como investigadora titular en 1995. Ingresó en la instituición de investigación Fundación Getúlio Vargas como profesora en 1982, y, en ella, Flery edificó una brillante carrera siendo impiedosamente despedida en marzo de 2018.

En función de su vasto legado a la Salud Colectiva, sus obras son de impacto profundo para pensar especialmente 'lo político' en la salud o que pude tener hecho su figura amenazadora en el contexto ultraliberal vivido actualmente. Así, en este estudio intentamos realizar un análisis, aúnque restrita a un periodo, de las matrices teóricas así como la contribución original que Fleury nos trae para pensar la economía política ${ }^{\text {vii }}$ de la salud. En 
este sentido es que nos concentraremos en el período de su intensa actuación en la construcción de la reforma sanitaria, es decir, entre 1989 y 1992 (ideario de la protección como derecho) considerado periodo nasciente de sua pensamiento.

Para ello, optamos por la modalidad textual de ensayo crítico, por la posibilidad de que la orientación no esté dada por la búsqueda de respuestas o enunciados verdaderos, sino por las preguntas que pueden orientar a los sujetos hacia reflexiones más profundas (ADORNO, 2003). Luego, el objectivo de este artículo fue realizar el análisis de dos de sus principales obras del período citado: Reforma sanitaria: "En busca de una teoría” de 1989 y "Estado y politicas sociales en América Latina" de 1992. Ambos textos tuvieron su confección en el contexto de la transición democrática en Brasil y en América Latina (postdictaduras militares), período este de conformación de la lucha por dentro del Estado en el post-constituyente en Brasil.

\section{Un sendero metodológico para una lectura sistemática de Fleury}

Para realizar esta tarea utilizamos un abordage cualitativo, con énfasis en la construción de un análisis de contenido sobre la teoría política de las principales obras de Sonia Fleury producidas en este período de crescimento sustancial de su pensamiento. En este sentido es que la consideramos una autora clásica de la Salud Coletiva, exactamente por generar un linaje de pensamiento político que perdura en el interior del campo. Como demuestra Brandão:

\footnotetext{
Existencia de estilos determinados, forma de pensar extraordinariamente persistente en el tiempo, modos intelectuales de relacionarse con la realidad que subsumen incluso los más lídimos productos de la ciencia institucionalizada, estableciendo problemáticas y continuidades que permiten situar y poner bajo nueva luz mucha propuesta política... (BRANDÃO, 2005, p. 236)
}

Durante estos cinco años (1989-1992), Fleury publicó veintitrés artículos científicos, en promedio de cuatro artículos por año, siendo seis de ellos en la Revista Salud en Debate del Centro Brasileño de Estudios en Salud (Cebes), trés en la Revista de Administración Pública de la Facultad Getúlio Vargas, entre otras. En cuanto a los libros, publicó cinco libros, siendo que dos de ellos se refieren a su producción más significativa.

Por lo tanto, realizamos el análisis de dos de sus principales obras del período citado: "Reforma sanitaria: En busca de una teoría" de 1989 y "Estado y politicas sociales en América Latina" de 1992. Ambos textos tuvieron su confección en el contexto de la 
transición democrática en Brasil y en América Latina (post-dictaduras militares), período este de conformación de la lucha por dentro del Estado en el post-constituyente en Brasil. Es importante aclarar que en realidad estas dos obras son compilaciones de artículos editadas por la autora (la de 1989 con dos artículos de su autoría, y la de 1992 con un artículo). Por lo tanto, no se analizarán las dos obras en su totalidad, sino los textos de Fleury que se encuentran en estas compilaciones. De un modo general, los artículos pueden ser considerados como una síntesis de algunos conceptos centrales del pensamiento de Sonia Fleury (sobretodo el concepto de ciudadanía) presentes en dos de los libros de la autora.

Mismo que dos obras (una de 1989 y la otra de 1992) aparezca como 'demasiado limitado' (sea en cuantidad - solo dos trabajos, o en temporalidad - el periodo de 1989 a 1992), especialmente cuando se tiene em mente el tamaño de la obra de la autora (más de 200 artículos y 26 libros, de 1977 a 2019), es esencial resaltar que estas dos obras eligidas son muy densas, además de estarem ubicadas en el tiempo histórico de nuestro análisis (transición democrática). Por eso, mismo que deseásemos la ampliación del corpus de análisis, creemos que era prudente limitarlo a un número o período específico para no caermos en una descontextualizión 'texto-contexto'.

Así, es importante dejar claro también que, mismo que se sepa que la autora detiene una obra literaria extensa, enfocamos en las dos obras anteriormente citadas porque ellas están contextualizadas en este marco histórico, o sea, sobre la constituición del Sistema Único de Salud mientras la transición democrática. En este período, la búsqueda por que se denominó como una 'teoría de la reforma sanitaria' impulsionó la autora a dedicarse a este tema. Además de este criterio, son estos dos libros que caracterizan la construcción teóricopolítica de la autora sobre el Estado, meslcando aspectos de teoría política e económica que dialogaban con el contexto vivido y con las posibilidades de interpretración sobre la acción concreta. Se hay alguna motivación para utilizar estos libros sino sua diversos artículos es justamente el rescate histórico de sus ricos fundamentos sobre el Estado, que, de manera general no viene siendo considerado en los debates de hoy.

El primer texto es producto de uno de los seminarios de investigación promovidos por el Núcleo de Estudios Político-sociales en Salud (NUPES) del Departamento de Administración y Planificación en Salud de la Escuela Nacional de Salud Pública (ENSP) en Brasil, en el contexto del proyecto de investigación "Políticas y estrategias de construcción del sistema de salud: perspectivas de la reforma sanitaria brasileña", que contó con la participación de investigadores como el Prof. Giovanni Berlinguer ${ }^{\text {viii }}$ y del Prof. Carlos Nelson Coutinho ${ }^{\text {ix }}$, realizado en el año 1989. Un enfoque más sistemático que exige 
su importancia en el contexto científico y político que vive la moderna sociedad brasileña (años 80 y período reciente post-constitución).

Ahí se introducieron las bases fundamentales para la comprensión del papel de las políticas de salud como proyecto sectorial de un Estado que, en una sociedad de clases, representa inequivocadamente los intereses de la clase dominante, sin dejar de presentar la posibilidad de expresión de los intereses contrahegemónicos en la aparente homogeneidad del género Estado. Se discute ampliamente en esta producción, las dificultades enfrentadas por la concepción histórico-estructural, que utiliza en el análisis de las cuestiones de la salud en el interior de la sociedad, apuntando, sin embargo, un elenco creativo de salidas metodológicas e hipótesis inovadoras que buscan explicar la emergencia del concepto de Reforma Sanitaria en un contexto democrático, a la luz de una perspectiva gramsciana.

El segundo texto es uno de los productos de los trabajos realizados en el Seminario "Salud y políticas sociales en América Latina" promovido por los NUPES en la ciudad de Río de Janeiro del 13 al 17 de agosto de 1990. Observase las transformaciones que el campo de la Salud Colectiva venía atravessando del punto de vista teórico-prático en las décadas de 1970 y 1980. Refierese a una reflexión en el análisis de las políticas de salud en la región.

Al abordar las dos obras de forma conjunta, apoyamos nuestro análisis en las dimensiones del análisis de contenido, tratadas por Quivy y Campenhoudt y Severino (QUIVY y CAMPENHOUDT, 1988; SEVERINO, 2002) o sea: las dimensiones temáticas con enfoque en la base evaluativa del contenido. Así, organizamos nuestra reflexión en tres partes. La primera presenta la síntesis del tema y problema propuestos por Sonia Fleury, identificando los conceptos y categorías (temática). La segunda parte busca construir el sentido estructurador de la base discursiva, identificando las matrices teórico-políticas utilizadas y su enfoque metodológico (base evaluativa). La tercera explicita la originalidad, validez, contribución teórica y histórico-política del autor.

Para Quivy y Campenhoudt, el análisis de contenido "se centra en mensajes tan variados como obras literarias, artículos periodísticos, documentos oficiales, programas audiovisuales, declaraciones políticas, actas de reuniones o informes de entrevistas mal dirigidas" (QUIVY y CAMPENHOUT, 1998, p. 226), y si en esta investigación teóricopolítica se eligió este método fue para privilegiar el estudio de las fuentes oficiales escritas (escritas por Fleury) como repositorios de su pensamiento. Así, esta técnica de análisis de datos "ofrece la posibilidad de tratar metódicamente información y testimonios que tienen cierto grado de profundidad y complejidad" (id., Ibid: 227), siendo utilizada, por tanto, en su hilo dedicado a discernir y descubrir el "sentido de los textos". Además, esta técnica de 
análisis es un valioso aporte para deconstruir tanto la parte retórica de los discursos oficiales de los textos como su parte programática, pues al fin y al cabo esta técnica permite tener en cuenta los más diversos aspectos formales del discurso "considerados indicadores de la actividad cognitiva del hablante, los significados sociales o políticos de su discurso o el uso social que hace de la comunicación" (id.).

Los corpora del texto fueron extraídos cuando la caregoía 'Estado' aparecía. Así, la discusión sobre el Estado como centro de un análisis de la economía política en el contexto de la Reforma Sanitaria Brasileña fue el guión del análisis. De esta manera, los extractos del texto fueron retirados como unidad de análisis para, a partir de ella reconstruir en sentido teórico que Fleury se utiliza desta esta categoría y también comprehender las matrices teórico-políticas que ella usaba en su tiempo social y histórico.

Por tanto, es pertinente ratificar que este estudio no se caracteriza por un 'análisis histórico' senso estricto de la 'pluralidad' de la Reforma Sanitaria ni una historiografía de 'gran angular'. Se considera que, de manera menos atrevida, se trató de una identificación textual localizada de esta autora-actriz en dos obras de relevancia histórico-política que narran sus percepciones sobre la Reforma de Salud debidamente sustentadas en una perspectiva marxista crítica. Por tanto, para asegurar la precisión analítica de lo emprendido en este trabajo, se trató de una comprensión inicial del análisis marxista utilizado por la autora-actriz del texto, y por el texto, sin pretensiones ampliamente inductivas a su contexto.

Finalmente, es fundamental enfatizar que el enfoque utilizado en este artículo no intenta considerar la historia como una 'maestra de la vida', incluso en desacuerdo con los métodos profesionales de hacer y comprender la disciplina histórica. Así, este estudio parte de la concepción de que la historia tiene funciones, y una de ellas es permitir la desnaturalización de estructuras y formas de pensar de diferentes épocas, posibilitando la redefinición de eventos y contextos, siempre dentro del ámbito de las teorías y formas de pensar específicas.

\section{EI Estado como centro del debate en Fleury: sus contribuiciones en la coyuntura de los 1980}

Sonia Fleury al proponer el estudio sobre la democracia y reforma sanitaria, en el contexto del período post-constituyente en Brasil, recurre a la perspectiva crítica inspirada en el análisis de la economía política marxista, con particular énfasis en el enfoque gramsciano, ampliándola con otras reflexiones teóricas, principalmente las que consideran 
el propio desarrollo de la ciudadanía en el Estado moderno estableciendo patrones de derechos sociales propios a cada nación. Esto queda explícito cuando Fleury argumenta que:

La simple introducción de la noción de intereses de clase no garantiza que el análisis político sobrepase el nivel de la reificación de las funciones de las políticas sociales. La cuestión del análisis de los determinantes de la intervención estatal pasa así a requerir una reflexión más exacta de la propia naturaleza del Estado, recuperando la noción básica de la contradicción y de sus manifestaciones históricas concretas. (FLEURY, 1989, p. 20)

Aunque la autora reconoce la importancia de la concepción histórico-estructural al área de la salud, como un nuevo paradigma de conocimiento de la relación entre medicinasociedad y consecuentemente del papel del Estado en esa relación - fortalecida por la articulación de los tres niveles económico, político e ideológico -, ella argumenta críticamente la reducción del análisis de esa concepción. La crítica de la autora recae al bajo grado de tratamiento de los niveles político e ideológico cuando utilizados de forma mecanicista, o sea, reduciendo toda la política social a un simples papel de reproducción del modo de producción capitalista en los niveles económico y/o superestructural. La autora está apoyada en la concepción de la visión marxista estructuralista, prácticamente con la gran influencia en aquel momento de Althusser. Esse autor fue representante del método de análisis estructuralista en la perspectiva marxista, particularmente después de la publicación de su libro, Por Marx (2005), con gran influencia en el mundo académico, incluso en Brasil, durante las décadas de 1960 y 1970.

Así, para Fleury el concepto de ciudadanía abarca el nivel del político de forma más completa. Para ella, "la ciudadanía es, al nivel político, la abstracción necesaria a la constitución, fundamento y legitimidad del poder político" (FLEURY, 1989, p. 20). Por su parte, Fleury argumenta que:

Si por un lado la ciudadanía como relación individual de derecho entre el ciudadano y el Estado, es la negación de la existencia de las clases sociales, por otro lado, su reconocimiento, contradictoriamente, fue imprescindible para la constitución, organización y lucha de las clases dominadas. (FLEURY, 1989, p. 21)

En esta perspectiva, al colocar la ciudadanía como una categoría fundamental en su análisis sobre la formulación para una Teoría para la Reforma Sanitaria Brasileña, especialmente en un país de la periferia del capitalismo, Fleury busca comprender la cuestión de la ciudadanía no sólo como espacio de consenso entre las clases pero principalmente como espacio de fortalecimiento de lucha y contrahegemonía de las clases 
subalternas. De esta forma, insiste Fleury

Así como el Estado capitalista es más que un instrumento de la dominación burguesa, debiendo ser comprendido en su naturaleza compleja, de la misma forma la ciudadanía es más que una mistificación de la relación de igualdad burguesa, siendo necesario comprenderla en su génesis y desarrollo. (FLEURY, 1989 , p. 21)

Una vez más, Fleury deja clara su idea de ciudadanía:

\begin{abstract}
Además de un conjunto de reglas formales de ejercicio del poder político, la democracia es el locus de articulación de las mediaciones entre Estado moderno y sociedad. La ciudadanía es, pues, la mediación que da organicidad a esta relación, en la medida en que, más allá o en negación a la fragmentación de las clases en la estructura social, articula el conjunto de individuo de una nación al Estado representativo, asegurándole la legitimidad necesaria para el ejercicio del poder. (FLEURY, 1989, p. 33)
\end{abstract}

En este aspecto, Fleury revela la base conceptual de la noción de ciudadanía, refiriéndose a la conclusión de O’Donnell y Schmitter (1986) - única referencia teórica al concepto de ciudadanía tratado por Fleury. Estos autores concluyen que: el principio central de la democracia y de ciudadanía, implica tanto "el derecho de ser tratado por los demás seres humanos como un igual en lo que se refiere a hacer elecciones colectivas y el deber por parte de los que implementan tales elecciones de ser accesible y responsable ante todos los miembros de la comunidad política" (FLEURY, 1989, p. 33).

Es fundamentalmente en este espacio de la conformación de la ciudadanía - y de las distintas ciudadanías - que Fleury reinterpreta el papel del Estado y de las disputas entre clases con intereses antagónicos en su interior, lo que debe requerir una ampliación de la concepción del Estado.

Sin embargo, a pesar de que Fleury afirma la necesidad del análisis de las disputas en el interior del Estado y de lucha por la construcción de una nueva hegemonía, ella resalta los aspectos de la selectividad estructural ${ }^{\mathrm{x}}$ del Estado capitalista y de los intereses generales de la clase dominante, lo que puede plantear dificultades concretas al problema del avance del proyecto reformista en la salud.

En esta línea, Fleury reconoce que los análisis relativos al proceso de formulación de políticas (con destaque a la política de salud) deben considerar la particular presencia del Estado capitalista brasileño, especialmente en la construcción del modelo de desarrollo nacional, basado en la conformación de una industrialización retardataria que impone características muy específicas y problemáticas a este desarrollo. 
Fleury argumenta acerca de esta especificidad cuando dice que:

El patrón de política social emergente en un contexto de industrialización retardataria fue marcadamente distinto de aquel que se originó en la liberaldemocracia. En primer lugar, hay un consenso sobre la necesidad de protección que atraviesa toda la sociedad, y en segundo lugar, el Estado es el mentor del proyecto y no los trabajadores. (FLEURY, 1989, p. 23)

Los resultados de este proceso permiten estructurar un sistema de protección social que tiene dificultades para orientarse por los principios de la universalización, sino por la "diferenciación de las categorías de trabajadores en relación a la pauta de beneficios a que tienen acceso" (FLEURY, 1989, p. 23). Además, Fleury pone énfasis en otras características de la limitación de nuestro capitalismo dependiente ante la construcción de políticas sociales más universalistas. Dice ella:

\footnotetext{
La diferencia de los países desarrollados, las economías atrasadas/dependientes se caracterizan por la incorporación de tecnología elevada y concentradora de capital, de forma abrupta y fragmentada, sin una articulación orgánica o un efecto expansivo para el conjunto de los sectores productivos. Esta modalidad desde el inicio monopolístico de organización de la producción tiene como característica central la heterogeneidad estructural de la economía con la concomitancia del proceso de expansión de las relaciones capitalistas y el mantenimiento, como partes integrantes de ese proceso de acumulación, de formas de inserción en la división social del trabajo no típicamente capitalistas [...]. De este complejo proceso de acumulación [emerge] una clase trabajadora altamente heterogénea, con diferentes formas de inserción en la producción. (FLEURY, 1989, p. 24)
}

Al reconocer la especificidad de nuestro Estado brasileño, economía y clase trabajadora, Fleury enfatiza la necesidad de considerar en el análisis de las políticas sociales los sistemas de representación y organización de intereses, las alianzas entre actores políticos, reivindicando una comprensión sobre los regímenes e instituciones políticas. En el contexto de la selectividad estructural del Estado, de forma más directa, Fleury sugiere que el análisis político de la seguridad social debe abarcar el entendimiento de la pauta orientada por los "grupos de presión" y por la "burocracia estatal", siendo que de manera jerarquizada de la actuación de los primeros sobre los segundos, basado en los trabajos de Mesa-Lago (MESA-LAGO, 1978). En esta cuestión, Fleury destaca su trabajo con Jaime de Oliveira (1986) - “(Im)Previsión Social: 60 años de historia de las previsionales en Brasil" - que estudia el comportamiento de la burocracia y de los grupos de intereses en los contextos políticos específicos de los países latinoamericanos, resaltando los "gobiernos populistas, los regímenes burocrático-autoritarios y los períodos de transición democrática”.

En ese marco de los contextos políticos latinoamericanos, que Fleury al referirse a 
los sectores dominantes, argumenta con base en trabajo clásico de Cardoso (1975) “Autoritarismo y democratización” (CARDOSO, 1975), que al proceder a la interrupción de los canales democráticos de representación de intereses, se presentan los "anillos burocráticos" que se constituyen formas de articulación de intereses involucrando la relación entre la burocracia pública y la privada (de la gran empresa nacional o internacional), permitiendo la penetración de los intereses privados en el interior de nuestro particular Estado.

Es importate aclarar que Fleury considera como burocracia pública los trabajadores públicos con estabilidad o no en sus funciones, pero que se encontran mas o menos fijos en vínculo de empleo con la administración pública. Ya la burocracia privada está relacionada a los trabajadores de servicios privados contratados por el Estado y/o que hacen actividades en su nombre, aún, también en esta categoría, están los funcionarios de las empresas privadas propriamente dichas que operan con el Estado con fines de garantizar los intereses de sus compañías. Así es que la idea de los 'anillos burocráticos', de acuerdo con Cardoso (1993), constituyen "un mecanismo por el cual se define implícitamente que la administración es suplementaria a los intereses privatistas y estos fluyen en sus relaciones con el Estado, a través de redes de complicidad personal. [...] En el caso de Brasil en el período nacional-populista (...) fue exactamente donde comenzaron a formarse las 'islas de racionalidad' de una tecnocracia planificadora" (CARDOSO, 1993, p. 99-100).

Es en el tratamiento dado a la cuestión del Estado, particularmente de nuestro Estado periférico capitalista, que podemos presentar los aspectos abordados por Fleury en el capítulo "Estado y Crisis: una perspectiva latinoamericana", de 1992, dando continuidad a las hipótesis relacionadas en su libro de 1989, sobre los análisis de las políticas públicas, en general, y de la salud en particular.

La búsqueda de la autora es comprender la crisis del Estado moderno y parte de advertencias metodológicas de la matriz de Poulantzas (1985), o sea, la crisis como acentuación de los elementos genéricos presentes y permanentemente en acción en un contexto contradictorio; especialmente en el contexto de la fase del capitalismo monopolista en vigencia hasta los años 1980, en que el nuevo papel económico del Estado implica relaciones nuevas entre la crisis económica y político-ideológica, revestándose entonces de un carácter estructural.

Este nuevo papel económico del Estado se presentó como un giro hacia aquella concepción que los socialdemócratas consideran como 'Estado Mínimo'. Con las política neoliberales, el avance del capitalismo bajo la reestructuración productiva post-fordista y la 
'revolución informacional' (con la crisis de agotamiento del petroleo exgiendo un cambio de la matriz energética) obligó el Estado a “contener” recursos. Pero, en un análisis marxista, el Estado no vino a ser 'mínino' pero se orientó al 'máximo' para defender las políticas neoliberales (que son hechas para permitir el avance de la socibilidad del capital), siendo por esta razón que, marxianamente hablando, el Estado no es el centro de la crisis ('crisis del Estado'), pero sí el centro es la caída de las tasas de las ganacias de las empresas capitalistas que exigen que el Estado de comprometa políticamente con la salvaguarda del capital.

Fue en ese escenario que la transición política (abertura democrática) del período militar (autoritario-populista) para la redemocratización en Brasil se configuró. El sistema de salud jugaba iun papel importante en este conflicto. El recién creado Sistema Único de Salud (SUS) estaba era objeto de negociaciones políticas (en la Asambla Constituyente) entre grupo de empresarios (especialmente de os complejos hospitalarios privados) que deseaban el liderazgo sobre las acciones y servicios de salud publica, mientras tanto, los sanitaristas (la cual Flery se adjucaba) y movimentos sociales querían un SUS público, estatal y principalmente universal. Bajo muchas dicusiones y intensas disputas, el SUS se juspositivó como público y universal, pero, con la apertura para la inciativa privada de manera complementaria. Esto fue un problema importante porque, como dicho, en este contexto del capitalismo neoliberal, el Estado estaba mucho inclinado a ceder las presiones privadas mismo que constitucionalmente hubiera hecho un compromisso legal con la demanda popular. Fleury argumenta que el entendimiento de la crisis debe estar apoyado en las transformaciones de la relación entre economía y política, acentuando que las intervenciones del Estado en la economía actúan como un factor que genera crisis económicas. Pero, en realidad, Fleury se utiliza del tema 'Estado en crisis' como instrumento teórico y metodológico para proponer una contraposición a los estudios europeos sobre el Estado y reforzar la visión de las particularidades de la relación entre el Estado y la sociedad en América Latina. De esta forma, posibilita la formulación de cuestiones específicas al Movimiento de la Reforma Sanitaria: ¿de qué forma la tradición marxista europea influenció la interpretación del Movimiento de la Reforma Sanitaria? ¿Cuáles son las especificidades de la relación entre sociedad y Estado burgués latinoamericano? ¿Cuál era la crisis permanente de representatividad? ¿Qué tipo de institucionalidad era posible?

La tesis central de Sonia Fleury, al atribuir énfasis a la particularidad del Estado en América Latina, procura dar cuenta de las continuidades y discontinuidades de la crisis 
política, actuando en las fisuras de un 'Estado de excepción'xi , poco permeable a la sociedad. En la práctica, este tipo de Estado acaba respondiendo a una situación de crisis económica y política del Estado de derecho democrático-burgués, impidiendo la consolidación de la forma democrática del dominio burgués.

De acuredo con Fleury, si esta propuesta es correcta, es importante reconocer que:

\begin{abstract}
... las especificidades del Estado capitalista periférico no sólo será importante para comprender su dinámica, distante de las hipótesis de una disfuncional edad en respecto al paradigma de la democracia, sino que aportará elementos para comprender las posibilidades de respuesta, incluso de los países industrializados y democráticos frente a las amenazas que genera la crisis económica. Es necesario recordar que tales amenazas deben ser percibidas a través de la lente del capitalismo monopolista que al alterar la relación Estado-política, delimita y reproduce el contexto de la crisis y de sus soluciones. (FLEURY, 1996, p. 29)
\end{abstract}

Así, Fleury busca responder si la crisis del Estado moderno es una crisis del Estado capitalista en sí, es decir, crisis de las relaciones posibles de organización política burguesa bajo el mando del poder capitalista. Al rescatar elementos relacionados a la transformación del Estado en Poulantzas (POULANTZAS, 1985), ella afirma que "éstos marcarían el fin de un ciclo virtual del capitalismo en que se desarrollaron de forma armónica la acumulación capitalista y la democracia sociopolítica, y que la crisis actual podría evidenciar la posibilidad de una nueva la separación entre capitalismo y democracia (FLEURY, 1992, p. 21).

Es importante añadir que crisis Fleury está hablando. Se trata del período que se agota a mediados de la década del 70, en el que se instala, progresivamente, lo que en otra ocasión se designó como 'crisis político-financiera' del modelo asistencial establecido en los años anteriores. 'Crisis', que en realidad no es más que una expresión sectorial de la situación más amplia, de crisis - a la vez económica y política, 'de legitimación' y 'físcal' - que atravesó el régimen autoritario, en esos años al final del período anterior de acelerado crecimiento económico y el inicio del llamado proceso de "apertura" política (OLIVEIRA, 1987).

Fue sobre este panorama que este aspecto de la difícil convivencia entre capitalismo y democracia ${ }^{x i i}$ constituye un problema que Fleury se ocupa desde el primer texto (1989), y que se recupera a partir de la visión de Przeworski (1989) en ese segundo texto de 1992. Para ese autor, el postulado del análisis marxista de la imposibilidad de conciliación entre el conflicto distributivo y la democracia bajo el capitalismo se mostró incorrecto. Para añadir a esta argumentación, Fleury se utiliza de Offe (1984), cuando evidencia cómo las 
experiencias democráticas tuvieron éxito dentro del modo de producción capitalista, en que coexistió los mecanismos responsables del sistema partidarista competitivo y el sistema de bienestar keynesiano, o es decir, la mercantilización de la política y la de la politización de la economía privada (FLEURY, 1992).

Así, con base en esos autores, Fleury insiste en la fragilidad del análisis marxista ${ }^{\text {xiii }}$ para explicar la naturaleza del Estado brasileño. Por su parte, el período específico del consenso keynesiano, aseguró la reproducción cotidiana del consenso activo en los grupos subalternos. De esta forma, Fleury (1992) destaca que no quedó predominante la lógica de la acumulación capitalista, posibilitando la inclusión de compromisos que contemplen la influencia pública sobre la inversión y la distribución de la renta.

Fleury destaca, sin embargo, que las crisis vivenciadas ponen en jeque nuevamente la cuestión de la convivencia pacífica entre democracia y capitalismo. Afirma que no hay acuerdo entre los autores sobre la relación del carácter estructural de la crisis y la pretendida irreversibilidad de las transformaciones impuestas a las formas de Estado, Gobierno y mediaciones entre Estado y Sociedad. Sin embargo, destaca que hay algún consenso sobre las transformaciones que ven ocurriendo, lo que abre un campo amplio para repensar las relaciones entre Estado y Capitalismo.

La autora, al abordar estas concepciones en distintas etapas del desarrollo capitalista, argumenta que su objetivo es: "demostrar la historicidad y singularidad de la compatibilidad entre la democracia como sistema de gobierno y el proceso capitalista de acumulación basado en la propiedad privada de los medios de producción” (FLEURY, 1992, p. 26).

De esta forma, la autora busca analizar las formas de Estado en latinoamérica, apoyándose en las características de esas estructuras políticas tratadas por Tilman Evers (1985) en "El Estado en la periferia capitalista". Se destacan entonces: a) la ausencia de organización plena de formas democrático-burguesas; b) ausencia de organización plena del Estado de Derecho; c) Funcionamiento deficiente del aparato del estado; d) concentración estatal de funciones económicas y políticas; e) Soberanía restringida.

En ese contexto, Fleury señala el paralelo que estas tendencias sistematizadas por Evers (1985) poseen con las formulaciones poulantzianas. En general, estas tendencias se expresan en los países de capitalismo periférico como una imposibilidad de establecer el Estado de derecho democrático-burgués, ante la constante presencia de elementos autoritarios y desestabilizadores, lo que vendría a conformar un Estado en crisis permanente.

A partir de las formulaciones de esos autores, Poulantzas e Evers, Fleury concluye que es fundamental el conocimiento de las especificidades del Estado capitalista periférico, 
con la intención de entender las posibilidades de respuesta para la consolidación de la agenda de derechos sociales en Brasil post-constituyente. En esa perspectiva, Fleury aclara:

El análisis del Estado en los países periféricos se beneficia de las 'ventajas del atraso' al romper con ciertas concepciones teóricas estigmatizadas e incapaces de explicar los aspectos cuestionados en la perspectiva actual mediante los siguientes recursos: abandonar la metáfora base-superestructura, introducir la heterogeneidad del económico, volver a defender lo 'político' como parte esencial de la esfera productiva y de la formación de los sujetos políticos, abrir espacio para analizar la construcción de la hegemonía y del carácter democrático de la lucha por el socialismo. De esta manera, se impediría que el Estado apareciera como derivación de la dinámica económica endógena o como epifenómeno. (FLEURY, 1992, p. 30)

La autora afirma que la formación social del capitalismo periférico y de la dominación burguesa tuvo como precedente no el mercado, sino la política en estas formaciones, y que la unidad social se dió a través del político, con fuerte presencia estatal y monopolizadora de la dinámica societal en su totalidad.

En este sentido, Fleury plantea al Estado como lugar privilegiado en el que se forman las clases y los actores sociales, afirmando que las fuerzas sociales no anteceden al Estado en estos casos, sino que se constituyen a partir de su intervención (punto central), dejando clara la idea de una gran autonomía otorgada al Estado. De esta forma, estas características serían responsables por el carácter tan personalizado de los intereses instaurados en la órbita estatal, ayudando a explicar la persistencia de prácticas clientelistas y patrimonialistas en la relación con organismos estatales y sectores sociales, expresando una lógica de negación de la representación (cuidadana) como principio estructurador de la arena política.

Las consecuencias políticas de estas características específicas de las formaciones latinoamericanas se expresan en la necesidad de la afirmación del Estado como un garante de la unidad nacional. En rigor, el poder político con el uso de la violência supre la incapacidad de las clases dominantes fragmentadas en construir el mercado y la unidad nacional. Esto mina las bases del dominio burgués al negar los principios de igualdad y libertad. Afirma que varios autores enfatizan que esta estructuración de las clases provocará consecuencias peculiares de tipo político, como la relación pueblo-movimiento nacional.

Para esta tarea, Fleury recupera formulaciones de Touraine (1976), Plaza (1984) y Laclau (1985) para caracterizar tal consecuencia política. De forma sintética, estos autores argumentan la extrapolación de una lucha entre clases antagónicas a partir de la categoría pueblo, a medida que las clases populares se constituyen como clases nacionales, tanto por su representación material, como por el contenido de sus representaciones colectivas y su 
horizonte histórico, de la burguesía la posibilidad del uso de la cuestión nacional como forma suya de consolidación de hegemonía.

De forma sintética, pasemos ahora al ítem sobre la identificación de las matrices teóricas.

\section{Las matrices teórico-políticas en Fleury y la originalidad teórica en su escena histórico-política}

Se trata de reconocer el intento de Fleury (de carácter pluralista), que, partiendo de la importancia del análisis marxista, procura ampliarla a la luz de una literatura que privilegia el análisis de lo político sobre lo económico en autores socialdemócratas de la ciencia política (latinoamericana e institucional - Przeworski y Offe). Conforme a la visión de esa autora sobre el pensamiento marxista (el de la visión estructuralista de base althusseriana $^{\mathrm{xiv}}$ ), insiste en su crítica, a medida que ella entiende ser esa vertiente despossuida del grado de tratamiento de los niveles políticos e ideológicos, a fin de permitir un mayor entendimiento del análisis de la reforma sanitaria en el período posterior a la constitución de 1988.

De ahí Fleury atribuye énfasis al concepto fluido de ciudadanía. Para ella: "las diferencias entre los diversos procesos de Reforma estarán en el contenido político de la propia concepción de ciudadanía y, por consiguiente, en la relación entre Estado/ciudadano" (FLEURY, 1989, p. 41). Para esta discusión Fleury no menciona el libro clásico de Marshall $^{\mathrm{xv}}$, sino de los autores de la escuela de la ciencia política latinoamericana, en la vertiente de la literatura sobre "Transiciones de los regímenes autoritários" de los años 1980 (O’DONNEL y SCHMITTER, 1986).

Así, Fleury en su análisis, parte de una matriz marxista, con base gramsciana, inclinandose sobre la historia específica brasileña y su forma propia de inserción especificidad de la economía (retardadora dependiente, con específica inserción en la división internacional del trabajo) y de la sociedad brasileña. De ahí, también, ella reconoce la importancia de la concepción de Gramsci respecto del Estado ampliado y de su estrategia de 'guerra de posición' ${ }^{\text {xvi }}$, construyendo un espacio del consenso en un ambiente democrático en el contexto de la sociedad civil más compleja, como la que Brasil presencia al final de los años 1980, al inicio del proceso democrático, marcado por la Constitución de 1988.

En realidad, en su crítica al pensamiento marxista, de vertiente estructuralista, busca 
retirar la fuerza de ese pensamiento, vigente en aquel momento en los análisis en el contexto de América Latina, ampliándolo con contribuciones de Poulantzas y Gramsci. Además, al destacar las categorías de los grupos de presión y de la burocracia estatal, Fleury se utiliza de la literatura institucionalista, con destaque para las contribuciones de Weber. Esto queda claro con el intento de Fleury de explicitar la importancia del análisis del aparato del Estado, resaltando el concepto de burocracia de Estado, en términos de racionalidad del Estado y la fuerza de grupos de presión en la definición de la pauta de las políticas públicas. En rigor, Fleury no hace mención a Weber, sino a sus seguidores, por medio de la utilización de autores como Fernando Henrique Cardoso (1975) (con la expresión de 'anillos burocráticos').

En síntesis, Fleury busca traducir la teoría marxista de base poulantizana y gramsciana a la condición histórica concreta de Brasil y esa es una cuestión esencial de su método. Su argumentación gravita en torno a que considera posible la transformación del Estado burgués sin su destrucción, por medio del sistema democrático y la organización de un poder político de nuevo tipo.

$\mathrm{Su}$ enfoque teórico-metodológico revela una investigación innovadora en el pensamiento social en salud por la perspectiva del Estado y del estructuralismo, que no se realizaban hasta entonces. Se vuelve su análisis para aspectos de la transformación del Estado Nación al Estado como actor político, controlado por una clase predominante burguesa que por medio de aparatos ideológicos (Althusser) y de la hegemonía o consenso hegemónico (Gramsci), explican en su totalidad las luchas de clases fuera del Estado.

En el análisis de la crisis del Estado contemporáneo, en los años 1980, ella reconoce la importancia que muchos autores socialdemócratas analizan la particularidad del Estado y la Democracia en el Capitalismo del occidente, apoyándose en Przeworski (1989) y Offe (1984) - autores que critican directamente la visión marxista.

Con base en la visión de esos autores sobre la crisis del Estado, Fleury aboga una lectura más específica al analizar la forma del Estado en América Latina, en el contexto del capitalismo periférico y tardío. De esta forma, procura contrastar con el análisis europeo del Estado dominado única y exclusivamente por la clase burguesa (TOURAINE, 1989; PLAZA, 1984; LACLAU, 1985). En ella, se destacan la especificidad de la lucha de clases en el Estado brasileño, que se aleja del análisis clásico entre el capital y el trabajo, pero mucho a partir de la revisión de Touraine (1976) por la categoría 'Pueblo' y la concpeción de las clases populares que se constituyen como 'clases nacionales', retirando de la burguesía la posibilidad de concluir la cuestión nacional consolidando su hegemonía. 
Es importante considerar la incorporación de Sonia Fleury por una lectura más amplia sobre el espacio de disputas en el interior del Estado brasileño, por medio de la adopción del concepto de 'ciudadanía'. Además, es necesario profundizar el entendimiento teórico sobre la construcción de la reforma sanitaria brasileña en el contexto de la democracia específica como la nuestra. La autora reconoce la particularidad de nuestro Estado brasileño y del proceso de modernización conservadora en que la política de salud y su reforma es desarrollada por el Estado y no por un proyecto de los trabajadores ${ }^{\mathrm{xvii}}$. De ahí la importancia de este análisis para entender el constante rechazo de los sindicatos en no profundizar la lucha de defensa por un SUS es explicativo, no por lo tanto, los trabajadores sindicalizados hacen mas la defensa de medicina prepaga específica para toda su categoria que un sistema público estatal.

Al tratar de la problemática de la conformación de los estados nacionales latinoamericanos afirma que tales Estados nacen de una contradicción básica estructural por cuenta de su nacimiento como necesidad de expansión económica de otros Estados-nación, lo que determinaría las funciones y el desarrollo de la nación, Estado y de las clases sociales en la historia de la región. Sin embargo, afirma que el desarrollo histórico de la estructura de clases en esta región es altamente compleja y heterógenea, no habiendo homogeneidad ni en la composición ni en los intereses de la propia clase dominante - parte articulada con los intereses del capital extranjero y parte articulada con formas precapitalistas de producción - mientras que en las clases medias y sectores dominados se ve la misma fragmentación, fruto de las distintas inserciones en la estructura productiva, así como de su incorporación al sistema político, lo que genera las conocidas formas de corporativismo estatal.

Sin duda, Fleury contribuye a una reflexión más amplia y macrosociológica sobre los problemas de la construcción de la reforma sanitaria en el contexto de democracia restringida y específica brasileña. Las hipótesis explicativas enunciadas en el artículo de 1989 buscan propiciar un status a la política de salud "no más ser concebida solamente como resultante, ya que se constituye en un espacio siempre específico de reproducción ampliada de las relaciones políticas y económicas”. Así, la comprensión de los fenómenos sociales se lleva a la idea de que un esfuerzo de la teoría sociológica contemporánea exigiendo que "en ser estructurado, es también estructurante de la realidad social" y (...) "necesita ser efectivamente incorporado a los análisis de las políticas públicas” (FLEURY, 1989, p. 29) caso los reformistas quieran la sustentabilidade del SUS en tipo de Estado y de democracia. 


\section{Consideraciones finales}

Quedámonos con algunos cuestionamientos para pensar como la economía política y sus categorias centrales como el Estado y el modo de producción capitalista podrían ayudarnos a comprender mejor el papel de las políticas de salud en pensamiento de Fleury. En que medida la producción actual en el campo de la Salud Colectiva abarca la amplitud del análisis formulado por Fleury, al articular Estado, capitalismo, democracia, ciudadanía, grupos de presión, burocracia estatal, y aún más, la particularidad histórica del Estado y de la clase trabajadora latinoamoriacana, la fragilidad de la cuestión de la nación y de una clase burguesa?

No cabe duda de que el espacio prioritario de reflexión de Fleury es el locus del Estado y no la discusión de la sociedad. Ella enfatiza la particularidad del Estado capitalista periférico en incorporar las demandas de la sociedad, los derechos sociales. Este el caminho de reflexión, aún incompleto, que Fleury no deja para pensar las políticas de salud.

En el marco de la inspiración de los análisis de Fleury, podríamos reflejar con mayor propiedad los problemas que se enfrentan a la construcción del SUS en la dinámica del capitalismo contemporáneo, con la conformación de un Estado cada vez más atravesado por el capital, principalmente por el capital que devenga intereses en Brasil. En ese sentido, sería importante reflexionar más sobre la tendencia de la salud pública universal en Brasil en su relación con el contexto de la crisis contemporánea del capital. De ahí, quizás incluso más amplio que Fleury, sería incorporar esa crisis a la visión de una crisis más profunda del capitalismo, que viene siendo desarrollada desde hace mucho tiempo.

El conflicto y la crisis son marcas fundamentales en la reproducción del sistema capitalista, y por cierto, ellos son constitutivos. El Estado no es indiferente a la reproducción económica de ese sistema capitalista siendo motor esencial para esa reproducción. La dinámica del capitalismo es que funda ese Estado moderno en el período de la acumulación primitiva de capital. Al referirse al capital como una relación social de producción, Marx lo sitúa muy bien como producto históricamente específico de determinada formación social y económica, en que el poderoso dominio político del Estado moderno aseguraba el proceso de expropiación violenta de la tierra (XXXX, 2015).

De acuerdo con Mascaro, “[...] el Estado se revela como un aparato necesario para la reproducción capitalista, asegurando el intercambio de las mercancías [...]”, en su formavalor “[...] y, la propia explotación de la fuerza de trabajo en forma assalariada" (MASCARO, 2013, p. 18). Así, se puede decir que el Estado, tomado a partir de su forma 
política, se revela con relativa autonomía a la totalidad social, explicitando así una separación entre el poder político y el poder económico (pero apenas en la aparencia). Sin embargo, tal autonomía se ejerce en el enmarañado de relaciones sociales particulares, es decir, relaciones capitalistas, de ahí la relatividad de su autonomía. Esta idea parece adaptarse mucho más al Estado contemporáneo brasileño. Claro que apartar la discusión que Fleury no es la salida, pero es necesario tenerla en cuenta de una manera crítica. Flery opta al abordar la visión marxista por una lectura que predomina el político, atribuyendo a una interpretación limitante al marxismo, es decir, desconociendo en hondo la crítica a la economia política hecha por él. La forma-valor es predominante en ese Estado y articulada con el papel político del capital, resta poco espacio para una lucha política más amplia "de ciudadanos" especialmente en contexto de superexplotación como en Brasil.

No se debe olvidar que en la constitución de la forma económica y de la forma política - y en el entrelazamiento de ambas -, permea necesariamente la historia, la lucha de clases y su movimiento. No significa que el Estado, si tiene autonomía ante las clases, no revela indiferencia en relación al todo social. En ese sentido, Mascaro (2013) es bastante claro: "no es el dominio del Estado por una clase que revela su razón estructural de ser; es la forma que revela la naturaleza de la reproducción social" (MASCARO, 2013, p. 45). Para reforzar el argumento ya explicitado anteriormente, hay una autonomía relativa del Estado que está anclada en la dependencia estructural y existencial de determinado tipo de reproducción social, la capitalista. Tratemos de insistir, es relativa porque refleja la propia reproducción capitalista. Mascaro sintetiza: "no es por las clases que lo dominan, sino por la forma, que el Estado es capitalista" (MASCARO, 2013, p. 50).

En esta perspectiva, cuando se refiere a la naturaleza de la crisis capitalista, es posible afirmar que el Estado desempeña un papel fundamental en su constitución, a medida que es forma necesaria de ese modelo de reproducción social capitalista.

Desde 1980 hasta el período actual, es decir, en los tiempos de vigencia del neoliberalismo, no fue posible identificar la retirada del Estado de la economía, sino al contrario, se asistió a una particular forma de "presencia" del Estado en la economía. En particular, en esta presente crisis del capitalismo, estamos asistiendo a la adopción de políticas ausentes por parte del Estado, con reducción de los derechos sociales, inclusive de la política de salud en el mundo. En particular, en Brasil, se observa el permiso del Estado a la apropiación del fondo público por el capital, así como sus crecientes recursos concedidos a la iniciativa privada, a través del aumento de las renuncias fiscales en el área de la salud, debilitando la capacidad de financiamiento del Sistema Único de Salud (SUS). 
Quizás este fenómeno sea demasiado complejo para ser mencionado solamente acá pero consideramos que lo mas importante que queríamos hacer fue esta incursión al pensamiento político de Sonia Fleury. Rescatar su persona y su aporte téorico es muy importante para no que se pierda la historia política sobre las categorías de la economía política de una lucha por el derecho a la salud en sociedades capitalistas como la nuestra.

\section{Bibliografía}

ADORNO, Theodore W. Notas de literatura. Rio de Janeiro: Editora 34, 2003.

ALTHUSSER, Loius. Por Marx. São Paulo: Editora Unicamp, 2005.

ARAÚJO, Angela Maria Carneiro; TAPIA, Jorge Ruben Bitón. Estado, classes e estratégias: notas sobre um debate. Crítica e Sociedade: revista de cultura política, Uberlândia, Brasil, v. 1, n. 1, p. 6-54, 2011.

AROUCA, Sérgio. O dilema preventivista: contribuições para a compreensão e crítica da medicina preventiva. São Paulo: Unesp: Rio de Janeiro: Fiocruz. 2003.

BRANDÃO, Gildo Marçal. Linhagens do pensamento político brasileiro. Dados. Revista de Ciências Sociais, Rio de Janeiro, Brasil, v. 48, n. 2, 231-269, 2005.

CAMPOS, Gastão Wagner de Souza. Saúde pública e saúde coletiva: campo e núcleo de saberes e práticas. Ciência \& Saúde Coletiva, Rio de Janeiro, Brasil, v. 5, n. 2, p. 219-250, 2000 .

CARDOSO, Fernando Henrique. As Novas Teses Equivocadas. In: CARDOSO, Fernando Henrique. Autoritarismo e Democratização. São Paulo: Editora Paz e Terra, 1975.

CARDOSO, Fernando Henrique. A construção da democracia: estudos sobre política. São Paulo: Siciliano. 1993.

DAGNINO, Evelina. ¿Sociedade civil, participação e cidadania: de que estamos falando? In: MATO D. Políticas de ciudadanía y sociedad civil en tiempos de globalización. Caracas: FACES, Universidad Central de Venezuela. 2004. p. 95-110.

DANTAS, André Vianna. Reforma Sanitária Brasileira: ainda em busca de uma teoria para 
um debate necessário. En Dantas, AV. Do socialismo à democracia: tática estratégia na reforma sanitária brasileira. Rio de Janeiro: Fiocruz; 2018. p. 149-203

EVERS, Tilman. El estado en la periferia capitalista. Siglo XXI, México, 1985.

FLEURY, Sonia. Estado y politicas sociales en América Latina. 1. ed. UAM - X/ENSP, 1992.

HARTO-DE-VERA, Fernando. Ciencia política y teoría política contemporáneas: uma relación problemática. Madrid: Editorial Trotta. 2006.

JÚNIOR, João Feres; POGREBINSCHI, Thammy. A Teoria da política. In: JÚNIOR, João Feres;

POGREBINSCHI, Thammy. Teoria Política Contemporânea - Uma introdução. Rio de Janeiro: Elsevier. 2010. p. 1-10.

LACLAU, Ernesto. Tesis Acerca de la Forma Hegemônica de la Política. En Labastida, Hegemonia y Alternativas Políticas en América. 1985.

MARSHALL, Thomas Humphrey. Cidadania, Classe Social e Status. Rio de Janeiro: Zahar. 1967.

MASCARO, Alysson Leandro. Estado e forma política. São Paulo: Boitempo. 2013.

MESA-LAGO, Carmelo. Social Security in Latin America: Pressure Groups, Stratification and Inequality. Pittsburgh: University of Pittsburgh Press. 1978.

MOTTA, Luiz Eduardo. A Favor de Althusser: revolução e ruptura na Teoria Marxista. 1. ed. Rio de Janeiro: FAPERJ/Gramma. 2014.

O'DONNELL, Guillermo; SCHMITTER, Philippe. Transitions from authoritarian Rules: Tentative conclusions about uncertain democracies. Woodrow Wilson International Center for Scholars between 1979 and 1981. Latin American Program. 1986.

OFFE, Claus. Problemas estruturais do Estado capitalista. Tradução de Bárbara Freitag. Rio de Janeiro: Tempo Brasileiro. 1984.

OLIVEIRA, Jaime A. Reformas e Reformismo: "Democracia Progressiva" e Políticas 
sociais (ou "Para uma teoria política da Reforma Sanitária"). Cad. Saúde Publica, Rio de Janeiro, Breasil, v. 4, n. 3, p. 360-387, 1987.

OLIVEIRA, Jaime; TEIXEIRA, Sonia Fleury. (Im) previdência social: 60 anos de história da Previdência Social no Brasil. Petrópolis: Vozes/ABRASCO. 1986.

OSMO, Alan; SCHRAIBER, Lilia Blima. O campo da Saúde Coletiva no Brasil: definições e debates em sua constituição. Saúde e Socidade, São Paulo, Brasil, v. 24, supl. 1, p. 205 $218,2015$.

PAIM, Jairnilson da Silva. Reforma sanitária brasileira: contribuição para a compreensão e crítica. Salvador: EDUFBA: Rio de Janeiro: Fiocruz. 2008.

PAIVA, Carlos Henrique Assunção; TEIXEIRA, Luiz Antonio. Reforma sanitária e a criação do Sistema Único de Saúde: notas sobre contextos e autores. História, Ciências, Saúde-Manguinhos, Rio de Janeiro, Brasil, v. 21, n. 1, p. 15-35, 2014.

PLAZA, Orlando. La Nación como Realidade y como Hipótesis: Aproximaciones Metodológicas. En VEGA. Teoria y Politico de América Latina. CIDE, México. 1984.

POULANTZAS, Nicos. O Estado, o poder, o socialismo. Rio de Janeiro: Edições Graal, 2a ed. 1985.

PRZEWORSKI, Adam. Capitalismo e Social-democracia. São Paulo: Cia das Letras. 1989.

QUIVY, Raymond; CAMPENHOUDT, Luc Van. Manual de Investigação em Ciências Sociais. Lisboa: Gradiva. 1998.

SEVERINO, Antônio Joaquim. Metodologia do trabalho científico. 22a ed. revista e ampliada. São Paulo. Cortez Editora. 2002.

TEIXEIRA, Sonia Fleury. Reforma sanitária: em busca de uma teoria. s.1, Cortez, 1989.

TOURAINE, Alain. Les Societés Dependantes - Essais sur L'Amerique Latine, Paris, Dumlot. 1976.

La crisis y las Transformaciones dei Sistema Político en América Latina. En:

Calderon, F. Socialismo, Autoritarismo y Democracia. Buenos Aires, Flacso. 1989. 
VILAR-RODRÍGUEZ, Margarita; PONS-PONS Jerònia. El debate en torno al seguro de salud público y privado en España: desde la transición política a la Ley General de Sanidad (1975-1986). Historia y Política, Madrid, Espanha, v. 39, p. 261-290. 2018.

XXXX, XXXX. XXXXXXXXXXXXXXXX. XXXXXX (USP. Impresso), v. 24, p. 66-81, 2015.

Recebido em: 03/08/2020

Aprovado em: 08/10/2020

Publicado: $1 \% 1 / 2021$

\begin{abstract}
${ }^{\text {i }}$ Professor Adjunto da Universidade Federal de São Paulo (UNIFESP) e é vice-coordenador do Programa de Pós-graduação em Ensino em Ciências da Saúde (PPGECS). Tem pós-doutorado em Saúde Pública (Ciências Sociais e Humanas em Saúde) pela Universidade de São Paulo (USP) em associação com a Universidad Nacional de La Plata (UNLP) e concluiu o doutorado em Saúde Pública (Política, Gestão e Saúde) pela mesma instituição em 2015.

ii Possui doutorado em Ciências Econômicas pela Universidade Estadual de Campinas - UNICAMP - (2005), livre-docência pela USP (2012) e pós-graduação em Política e Relações Internacionais pela Lancaster University da Inglaterra (1984). Atualmente é professor livre-docente de economia política da saúde da Faculdade de Saúde Pública da USP e de seu programa de pós-graduação em Saúde Pública, professor doutor do Departamento de Economia e do Programa de Pós-Graduação em Economia Política da Pontifícia Universidade Católica (PUCSP). Professor convidado da Queen Mary, University of London, 2017 e 2019. É pesquisador Nível 2 de Produtividade do CNPq.

iiiEl campo de la Salud Coletiva en Brasil no es lo similar a lo que se conoce como Medicina Social en los países latino-americanos o, aproxidamente, lo que es Public Health/Salud Pública en los países anglo-sajones y ibéricos, pero mucho mas que esto. La Salud Colectiva es un aporte teórico-político cuyos orígenes se encuentran a fines de la década de 1970, en un contexto en el que Brasil vivía una dictadura militar. De acuerdo con Osmo y Schraiber (2015), la Salud Colectiva nació en este período, vinculada a la lucha por la democracia y al movimiento de Reforma Sanitaria. Las influencias del preventivismo y la medicina social se señalan en su constitución. Campos (2000) define la Salud Colectiva como un campo de saberes y prácticas sobre la salud tomándola como un proceso socialmente determinado. Así, este campo presenta tres grande áreas que se entrelazan: la 'epidemiología', la 'política, planificación y gestión en salud', y, las 'ciencias sociales y humanas en salud'.
\end{abstract}

${ }^{i v}$ Como dicho anteriormente, la Salud Colectiva en Brasil no es, por consequencia, un producto de acciones de apoyo a pesquisas en salud apenas, mas sí um gran movimento de lucha política con um cuerpo teórico construído para el enfrentamiento político. Sin embrago, no se puede negar la contribuición que Sonia Fleury tuvo para construcción de la teoría necesária para consolidar el campo en Brasil.

"Principal líder de la Reforma Sanitaria en Brasil que dió cuerpo teórico-político a lo que hoy es el Sistema Único de Salud (SUS). Por supuesto que Sergio Arouca fue uno de los grandes académicos involucrados en el Movimiento de Reforma Sanitária en Brasil, pero es difícil atribuir a qualquer persona el título de "principal responsable" por un gran movimiento social, político e histórico como este. Para Paiva y Teixeira (2014), sí, es preferible trabajar con la ideia de 'autores-actores'.

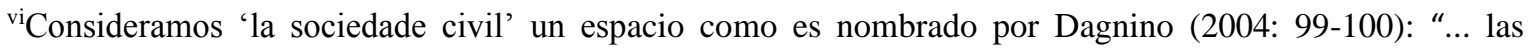
nociones de sociedad civil, participación y ciudadanía mantienen una estrecha relación entre sí y fueron seleccionadas porque son, desde nuestra perspectiva, elementos centrales de este desplazamiento de significados que constituyen el mecanismo privilegiado en la disputa política que se desarrolla hoy en torno al diseño democrático de la sociedad. [...] Esta centralidad, por un lado, está relacionada con el papel que jugaron en el origen y consolidación del proyecto participativo. Por otro lado, y por tanto, son fundamentales precisamente porque constituyen los canales de mediación entre los dos campos ético-políticos". 


\begin{abstract}
viïEl Estado es uma categoria central tanto para la 'ciencia política' cuanto para la 'economia política'. Mientras tanto consideramos que Fleury hace más un análisis 'economia política' en su obra que un análisis de 'ciencia política' como se considera este campo hoy. Actualmente la ciencia política tiene fuerte influencia cuantitativa, positivista y institucional - muchas veces reduciéndola a la administración pública (Harto-deVera, 2006). Además la ciencia política actual refuerza uma supuesta 'neutralidade axiológica' (Júnior y Pogrebinschi, 2010) que, em nuestro punto de vista no existe. Así, mismo sabendo que su formación fue en um posgrado de ciencia política dicho 'tradicional' sua trabajo tende más a un análisis de economia política por alargar la discusion del político para mas allá del Estado y considerar el movimento del capital como uma base material de determinación relativa importante sobre el Estado.
\end{abstract}

viii Médico sanitarista, bioeticista, diputado, senador, miembro del parlamento europeu, inspirador de la reforma sanitária brasileña, militante del Partido Comunista Italiano y gran activista del derecho a la salud en Brasil.

${ }^{i x}$ Uno de los politólogos marxistas más reconocidos en Brasil. Principal especialista en el trabajo de Antonio Gramsci en el Brasil y en el exterior. Fue profesor de teoría política en la Escuela de Trabajo Social de la Universidad Federal de Río de Janeiro.

‘'Selectividad estructural del Estado' es un concepto relacionado al esfuerzo de pensar en los mecanismos internos al funcionamiento del Estado, responsables de determinar el contenido capitalista de las políticas de Estado (incluso ahí, las políticas públicas de salud). Hay un debate en el interior de la teoría social marxista sobre, precisamente, los posicionaminetos de Claus Offe. Offe parte de la crítica a las posiciones presentes en el debate Miliband-Poulantzas, cuestionando la consistencia de las teorías de la "influencia" (Miliband) y las teorías de las "coacciones" o "factores limitantes" (Poulantzas). Las teorías de la "influencia" (Offe se refiere tanto a autores marxistas como pluralistas) serían las que tienen una visión instrumentalista del Estado, atribuyendo su carácter de clase a la influencia que ejercen directamente los intereses capitalistas dentro del aparato estatal (ARAÚJO y TAPIA, 2011).

${ }^{x i}$ En este momento, mientras, 1989 y 1992, Brasil había recién salido de un doloroso régimen de dictadura militar cuya parte más grande de los analistas consideraban haber sido un Estado de excpeción en relación a la democracia.

xiiEn un análisis marxista, capitalismo y democracia son temas que surgen como antagónicos. Es mucho comum que alguns analistas se pregunten: Si la democracia y el capitalismo tienen una difícil convivencia, por qué, entonces, la estrutura capitalista sostiene los regímenes democráticos? La contesta marxista para esa pregunta está relacionada con la comprehension sobre que se quierse decir con 'democracia'. La democracia que "convive" con el capitalismo es la 'democracia burguesa liberal', o sea, una manera procedimental de elección de soberanos y algunas liberdades democráticas en relación a la detención de la propiedad y su sucesión (herencia) sostenida por la noción universal de 'igualdad jurídica'. Los marxista critican esa democracia burguesa, diciéndole que democracia no se restringe apenas a eso. La democracia esta relacionada a la participación de los trabajadores mas amplia posible en las funciones políticas (democracia directa). En ese sentido, los marxistas apuntan que la democracia real solamente se puede vivir en una sociedade comunal (comunista) y que lo que se vive en el capitalismo es un simulacro democrático producido historicamente para justificar el papel "neutral" del Estado adelante de la relación capital-trabajo y que la 'representación' oscurezca los intereses de los representados impidiéndoles de dar dirección popular a las políticas. Este último aspecto restringe lo político a lo institucional.

xiiiLa cuestión es que Fleury parte de un análises marxista mas que, con esta crítica téorica, desarrolla un dibujo táctico-político socialdemócrata. El primero argumento se relaciona al hecho de que la experiencias que Przeworski y Offe desconsideran la ideia de democracia puesta en la nota de pié de página 9. Además, los autores (un polaco y otro alemán, hablan del centro capital) y no tuvieron como objeto los países de la américa latina en sus análisis, así no necesitaron hacer una mediación con la categoria 'dependencia' economica latinoamericana em un mundo ya ordenado por el imperio capitalista. Por ende, parte más grande de la izquierda estaban convencidos que la coyuntura política de la dictadura en Brasil juntamente con la crisis del capital (interna y externa) no debaja margen para una lucha con armas. Así, mismo que la radicalidade teórica hubiese puesta en el debate, la salida práctica (impelida por la situación coyuntural) era institucional (reformas por dentro del Estado - socialdemócrata). 


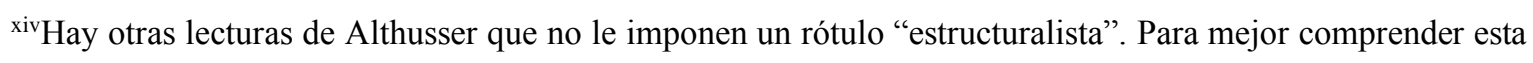
perspectiva váease Mota (2014).

${ }^{\mathrm{xv}}$ Viene de Marshall en "Cidadania, Classe Social e Status" el conpecto original de democracia. No creemos que la autora desconozca el concepto originário. Puede ser que ella hubiera eligido estos autores (O'Donnell y Schimitter, 1986), por la tradicción en las ciencias de la salud en trabajar con citas de autores más contemporaneos.

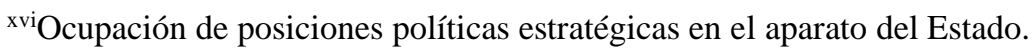

xviiParece contradictorio decir que hay una tensión entre Estado y democracia para después decir que el SUS y la Reforma Sanitária son projectos desarrollados por el Estado sino por la clase trabajadora. Entonces por qué el Estado, que reproduz los intereses burgueses, caminaría con una gran reforma sanitaria? Porque el Estado y sus agentes tuvieron que ceder en face a la presion organizada del movimento de los reformistas, especialmente fuerte em aquel momento donde las camadas medianas y populares apoyaban los cambios democráticos de apertura política y el SUS era visto como parte de estos cambios. Por outro lado, el avance de las fuerzas neoliberales de apropiación privada del fondo público a partir de la década de 90 empezó a ver en el SUS oportunidades de negócios. Esto eran tanto en subfinanciar sistema (descalificando su estrutura y forzando las camadas medianas a acceder a los servicios privados, desencorajando el uso público) así como con la gerencia privada de los servicios públicos y también la compra de servicios privados asistenciales para "completar" lo que faltava en la estrutura pública de servicios con alto grado de incorporación tecnológica (ya que laconstituicion no prohibe la participación complementaria del privado al sistema). 